\title{
Über die therapeutische Bedeutung der v. Pirquetschen Impfung.
}

\author{
Von \\ Dr. med. Wilhelm Mïnch, \\ Assistenzarzt.
}

Soweit mir die Literatur bekannt, ist bisher nur die diagnostische Bedeutung der kutanen Tuberkulinreaktion berücksichtigt worden. Ihren therapeutischen Wert zu prüfen, veranlassten mich teils eigene Beobachtungen, die ich im Frühjahr 1908 gelegentlich zahlreicher Tuberkulinimpfungen an der Giessener Kinderpoliklinik machen konnte, teils die Publikationen von Holländer und Joseph. Diese Autoren fanden, dass gewisse Formen von Hauttuberkulose Tuberkulose anderer Organe, insbesondere der Lunge, nahezu ausschliessen, ein Befund, der ohne weiteres zu denken gibt. Unwillkürlich drängt sich die Frage auf: Handelt es sich bei diesen Erscheinungen um reine $\mathrm{Zu}$ fälligkeiten oder lässt uns der Körper einen Einblick in den komplizierten Mechanismus seiner Schutzvorrichtungen tun? Kann man vielleicht durch Anlegung künstlicher Hautherde, die zwar in ihrem äusseren Anblick und histologischen Bilde den sogenannten toxischen Tuberkulosen ausserordentlich ähneln, jedoch der lebenden spezifischen Erreger entbehren, eine günstige Beeinflussung echter Tuberkulose herbeiführen? Was lag näher als die v. Pirquetsche Kutanreaktion hierfür zu benutzen. Haben doch bekanntlich manche Reaktionsformen der v. Pirquetschen Impfung eine täuschende Ähnlichkeit mit den sogenannten Hauttuberkuliden.

Die Versuchsanordnung gestaltete sich etwa folgendermassen:

Vor Beginn jeder Impfkur wurde ein genauer Status aufgenommen und, wenn irgend möglich, das Gewicht bestimmt. Desgleichen 
wurden genaue Temperaturmessungen vorgenommen, im allgemeinen morgens und abends; bei Patienten mit unregelmässigen Temperaturkurven auch mittags, bisweilen dreistündlich. Es wurde auf das Aussehen, die Farbe und Menge der verschiedenen Exkrete der Kranken geachtet, bei Lungen- und Nierentuberkulose insbesondere auf Bazillenreichtum und Lymphozytenmenge, bei letzterer ausserdem auf den Albumengehalt des Urins.

$\mathrm{Zu}$ allen Impfungen benutzte ich das Koch sche Alttuberkulin aus den Höchster Farbwerken, das sich stets gut bewährte.

Das Instrumentarium, dessen ich mich stets bei der Ausführung der Impfung bediene, ist sehr billig und denkbar einfach. Der Impfbohrer besteht aus einem, an seinem freien Ende breit geschlagenen, in eine Metallhülse gefassten, $2 \mathrm{~cm}$ langen Platindraht. 7um Auftragen des Impfmaterials nehme ich ein Glasstäbchen, wie es aus der Ophthalmologie bekannt ist. Das eine Ende wird rauh gefeilt, um möglichst kleine, annähernd gleich grosse 'Tropfen zu erhalten. Die Tropfen lasse ich an der Skarifikationsstelle eintrocknen, was bei Kindern kürzere, bei Erwachsenen längere Zeit beansprucht, höchstenfalls 1 Stunde. Legt man auf ganz gleiche Versuchsbedingungen Wert, so muss eigentlich die Haut zuvor gleichmässig durchblutet sein, was sich in einem lauwarmen Bade leicht erreichen lässt. Über die Ausführung der Impfung ist zu sagen, dass man das Instrument innerbalb der Tuberkulintropfen etliche Mal mit Hilfe der 3 ersten Finger der rechten Hand rotieren Jässt, wobei jeder stärkere Druck unter allen Umständen zu vermeiden ist. So erhält man eine ganz oberflächliche Hauterosion, die bei geschickter Ausführung nicht bluten und keine Narbe zurücklassen soll. Selbstrerständlich wurde vor jeder Impfung Instrumentarium und Operationsfeld gut gereinigt. Der Platindraht wurde in der Flamme ausgeglïht, Glasstäbchen und Haut mit Spiritus oder Äther desinfiziert.

Um einen möglichst genauen Einblick in die etwaigen Bedingungen für die therapeutische Wirksamkeit der Kutanreaktion zu erhalten, gestaltete ich die Versuchsanordnungen hinsichtlich der Zahl der Impfstellen auf der Körperhaut, der Zahl der Impfpunkte und der Daver der Pausen zwischen den einzelnen Impfungen recht mannigfaltig. Als Ort für die Impfung wählte ich bald die Brust-, Bauch-oder Rückenhaut, bald die Haut der Extremitäten sowie des Halses. Das eine Nal impfte ich in der Nähe des Krankheitsherdes <z. B. eines tuberkulösen Gelenkes), das andere Mal weit davon entfernt.

Die Zahl der Impfpunkte war bei den einzelnen Fällen recht verschieden. Meist fing ich mit 2 an, um nach Ablauf der ersten Reaktion bei einem Teil der Patienten die Anfangszahl bei- 
zubehalten, bei einem anderen langsam höher zu steigen, bei einem weiteren sprungweise vorzugehen. Die höchste Anzahl Impfpunkte, die in einem Falle ein Patient erhielt, betrug 90.

Wiederholt wurde die Vakzination gewöhnlich dann, wenn die eigentliche Reaktionsröte geschwunden war. Meistens war dies nach 5 bis 8 Tagen der Fall.

Der Verlauf der Reaktion bei mehrmaliger Impfung gestaltet sich ähnlich wie bei einmaliger Pirqeutierung. Auffallend war nur in vielen, vor allem den stark reagierenden Fällen eine verschieden grosse, etwa $1 / 2$ bis 2 Stunden anhaltende Flächenröte um die Impfpunkte, die eine urtikarielle Blässe zeigten. Sobald diese Erscheinung verschwunden war, waren die Anfänge der eigentlichen Reaktionsröte zu bemerken. Die nächste Umgebung der geimpften Stelle schwoll an und rötete sich. Alle Stadien der Entzündung konnte die Papel durchlaufen. Injektion, Infiltration, Exsudation mit Quaddelund Pustelbildung. Bei den heftigsten Reaktionen dokumentierte sich die grössere Exsudation durch urtikarielle Blässe des Zentrums, das ähnlich wie die umgebende Haut gefärbt und von einem hellroten Ring umsäumt war. Gewöhnlich stand die Impfpapel nach 3 bis 5 Tagen auf der Höhe ihrer Ausbildung, um dann rasch oder erst allmählich wieder zu verschwinden.

Ausser diesen rein lokalen Veränderungen an der Impfstelle selbst kam es nicht selten, je nach der Yersuchsanordnung, zu Störungen des Allgemeinbefindens und Herdreaktionen, auf die ich gleich zu sprechen kommen werde. Bei dem positiven Ausfall der Reaktion unterschied ich 2 Grade:

1. I. Grad der Stärke $=+$ Papel, 2 bis $5 \mathrm{~mm}$ breit, innerhalb 5 bis 6 Tagen verschwindend.

2. II. Grad der Stärke $=++$ Papel 5 bis $10 \mathrm{~mm}$ breit u. m., erst nach 6 Tagen oder später verschwindend.

Von den 24 therapeutisch Geimpften litten 9 an Lungen-, 7 an Knochen-, 4 an Lymphdrüsen-, 3 an Gelenk- und 2 an Nierentuberkulose sowie 2 an suspekter chronischer Bronchitis. Tuberkelbazillen waren in 9 Fällen nachweisbar. Bei den Lungenkranken unterschied ich 3 Stadien:

1. Stadium: Spitzenaffektion mit und ohne Fieber.

II. Stadium: Infiltration der Ober- und übrigen Lappen.

III. Stadium: Progresse Fälle mit Kavernenbildung, hektischem Fieber und Kachexie. 


\begin{tabular}{|c|c|c|c|c|c|c|c|}
\hline 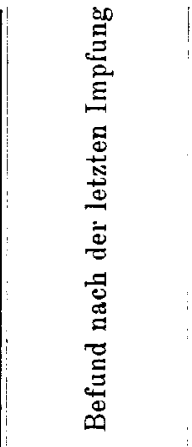 & 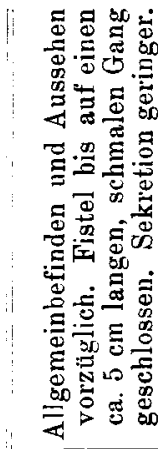 & 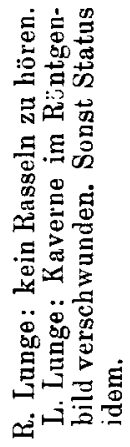 & 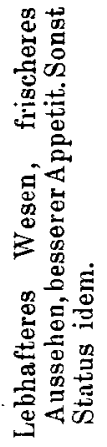 & 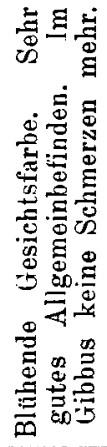 & 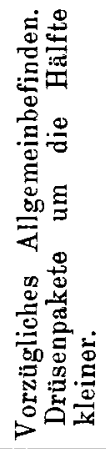 & 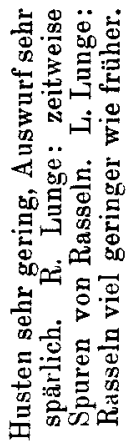 & 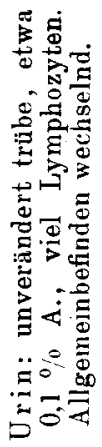 \\
\hline 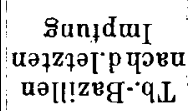 & & $\frac{x}{\frac{\pi}{5}}+$ & - & & & + & 1 \\
\hline q[ยบ.เәuน! & 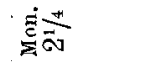 & $\frac{\pi}{\sigma_{0}}$ & $\overline{\bar{m}}$ & $\frac{+1}{m}$ & $\frac{+}{n}$ & $\frac{\pi}{\sigma 0}$ & os \\
\hline 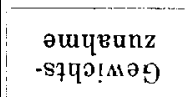 & कृषे & & $\frac{1}{\infty}$ & 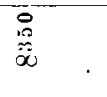 & 吕 & 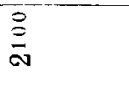 & 1 \\
\hline 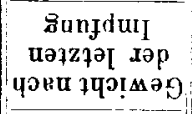 & $\stackrel{8}{g}$ & $\stackrel{8}{\stackrel{\Xi}{\Xi}}$ & 品 & $\begin{array}{c}0 \\
0 \\
20 \\
01 \\
01\end{array}$ & 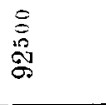 & $\stackrel{\varrho}{0}$ & $\mathscr{B}$ \\
\hline 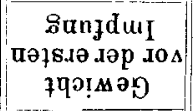 & 我票 & $\stackrel{N}{ت}$ & 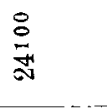 & 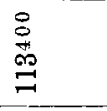 & $\stackrel{\infty}{\infty}$ & 5 & $\stackrel{\circ}{8}$ \\
\hline 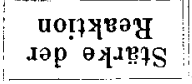 & $\begin{array}{l}+ \\
+\end{array}$ & + & + & $\begin{array}{l}+ \\
\vdots \\
+\end{array}$ & $\begin{array}{l}+ \\
+\end{array}$ & $\begin{array}{l}+ \\
+\end{array}$ & + \\
\hline $\begin{array}{l}\text { uәsunfduI } \\
\text { xәp IqeZ }\end{array}$ & 12 & 90 & $\stackrel{ }{\circ}$ & 0 & $\sigma$ & $\sigma$ & $\sigma$ \\
\hline 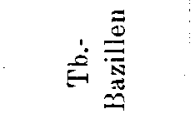 & & 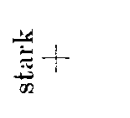 & & & & $\frac{a}{\stackrel{\vec{z}}{\infty}}+$ & $\frac{x}{\frac{\pi}{5}}+$ \\
\hline 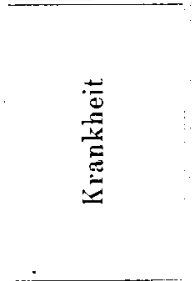 & 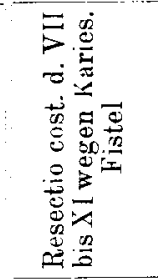 & 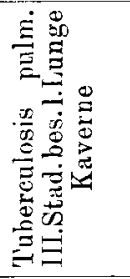 & 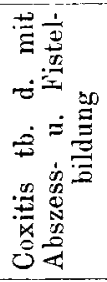 & 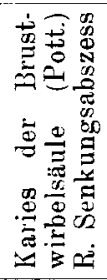 & 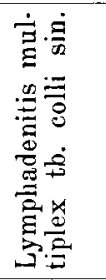 & 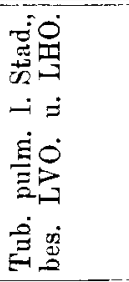 & 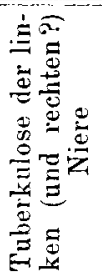 \\
\hline $74 ว \partial[40520$ & $\Xi$ & $\Xi$ & $\Xi$ & 3 & $\Xi$ & 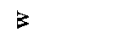 & $\geqslant$ \\
\hline IOHIV & 8 & $\underset{\sim}{\infty}$ & $\cos$ & $\not$ & $\stackrel{\varrho}{\varrho}$ & $\stackrel{\infty}{=}$ & $2 \pi$ \\
\hline 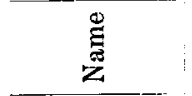 & $\stackrel{5}{3}$ & 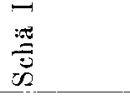 & $\stackrel{2}{2}$ & $\sum_{z}$ & 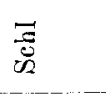 & \pm & 2 \\
\hline$\dot{\check{Z}}$ & . $\rightarrow$ & ov & SO & H & 25 & $\infty$ & 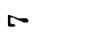 \\
\hline
\end{tabular}


5] Über die therapeutische Bedeutung der v. Pirquetschen Impfung.

263

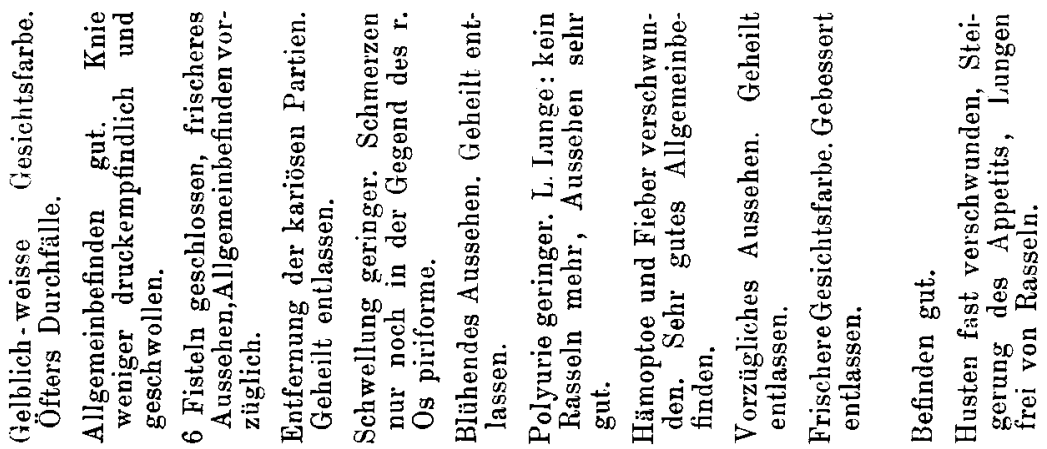

\begin{tabular}{|c|c|c|c|c|c|c|c|c|c|c|c|}
\hline$\stackrel{\vec{I}}{\vec{\nabla}}$ & $\neg$ & $\stackrel{*}{=}$ & - & $\doteq$ & \pm & $\stackrel{\Delta}{*}$ & $\stackrel{2}{\Rightarrow}$ & $\stackrel{x}{=}$ & $\stackrel{1}{=}$ & $\stackrel{21}{\Rightarrow}=$ & \\
\hline-1 & $r$ & $\frac{\bar{\sigma}}{\partial}$ & $\frac{\partial}{\sigma}$ & & $\stackrel{\mathscr{E}}{\circ}$ & $\sigma$ & $\begin{array}{c}\overline{\overline{0}} \\
\hat{\sigma}\end{array}$ & $\frac{3}{\pi}$ & $\stackrel{\partial}{\stackrel{\partial}{+}}$ & $\psi$ & 惫 \\
\hline 9 & $\stackrel{5}{\vec{y}}$ & 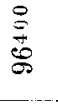 & $\frac{8}{\frac{8}{8}}$ & I & $\stackrel{0}{a}$ & 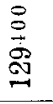 & $\begin{array}{l}\stackrel{8}{\circ} \\
\stackrel{\leftrightarrow}{\infty} \\
\stackrel{-1}{0}\end{array}$ & 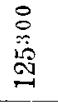 & 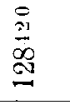 & $\stackrel{90}{=}$ & 言 \\
\hline$F$ & $\mathscr{H}$ & 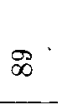 & 5 & 1 & 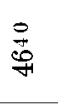 & $\begin{array}{l}\stackrel{\Xi}{\Xi} \\
\text { N̦ }\end{array}$ & $\mathscr{\rho}$ & $\begin{array}{l}\overline{\bar{O}} \\
\hat{\hat{\lambda}} \\
\stackrel{0}{=}\end{array}$ & $\Xi$ & $\exists$ & $\stackrel{\Xi}{\bar{\Sigma}}$ \\
\hline+ & + & + & + & + & + & $\begin{array}{l}+ \\
+\end{array}$ & + & + & + & + & + \\
\hline$\Sigma$ & $r$ & $\infty$ & $\omega$ & $\infty$ & $\infty$ & + & $\vec{r}$ & $\infty$ & ه & $\infty 0$ & هo \\
\hline & & & & & & & $\begin{array}{l}\frac{3}{0} \\
\stackrel{\pi}{E}+\end{array}$ & & 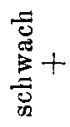 & & 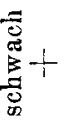 \\
\hline
\end{tabular}

\begin{tabular}{|c|c|c|c|c|c|c|c|c|c|c|}
\hline 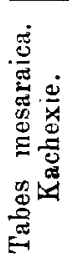 & 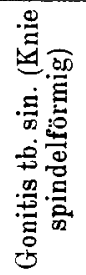 & 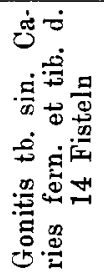 & 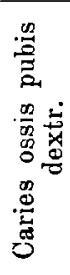 & 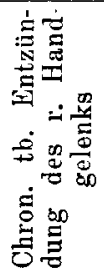 & 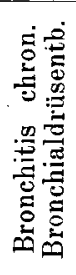 & 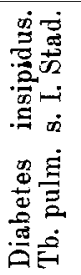 & 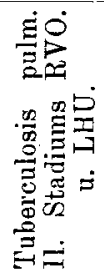 & 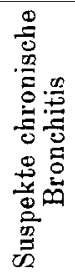 & 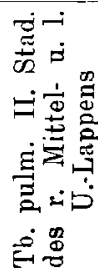 & 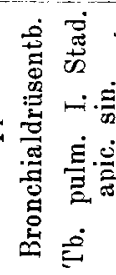 \\
\hline$\Xi$ & $\geqslant$ & $\Xi$ & $\xi$ & $B$ & $\xi$ & $E$ & $\Xi$ & $\geqslant$ & $\Xi$ & 3 \\
\hline 0 & $\infty$ & $\vec{\infty}$ & $\widehat{\widehat{\sigma}}$ & 荤 & 음 & $\mathscr{\rho}$ & $\tilde{\infty}$ & 8 & 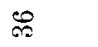 & 20 芯 \\
\hline
\end{tabular}
苟

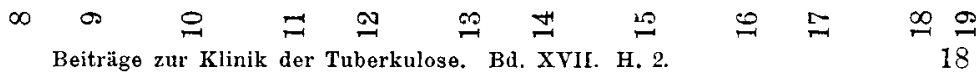




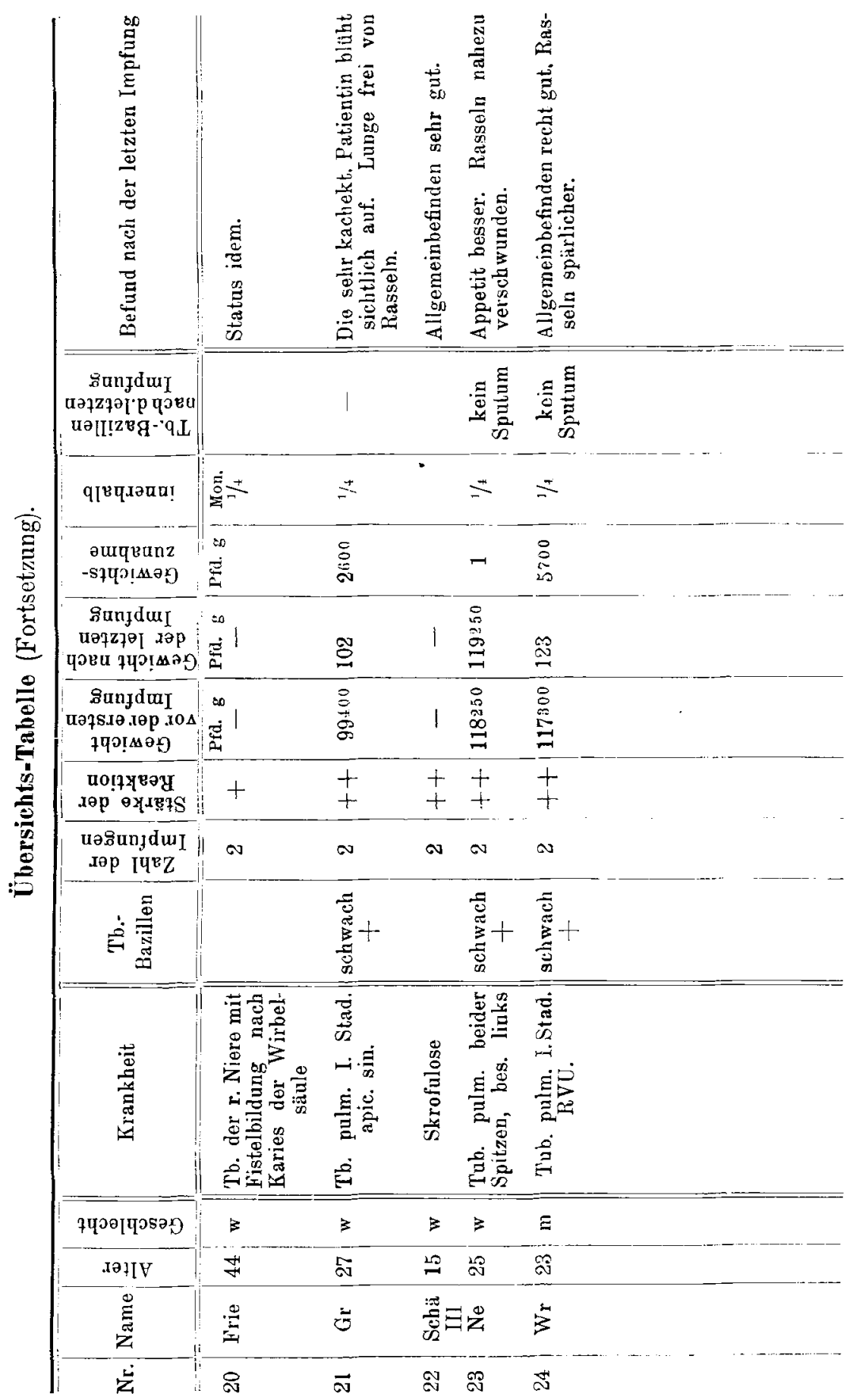


Dem I. Stadium gehörten 5, dem II. 3, dem III. 1 Kranker an. Ein Fall war kompliziert durch Diabetes insipidus. Die Knochenund Gelenkserkrankungen, die mit Impfungen behandelt wurden, waren teils leichter, teils mittelschwerer und schwerer Natur. Bei einer Patientin mit Nierentuberkulose fanden sich anfangs bei jeder Untersuchung Haufen ron Tuberkelbazillen im Urinzentrifugat. Bemerkt sei noch, dass neben der Impfung selbstrerständlich eine sachgemässe Behandlung der einzelnen Fälle durchgeführt wurde. Um eine rasche Orientierung über die mit der r. Pirquetschen Impfung erzielten therapeutischen Erfolge zu ermöglichen, habe ich in anliegender Tabelle kurz alles Wesentliche aus den einzelnen Krankengeschichten zusammengestellt. Einen Einblick in die verschiedenen Versuchsanordnungen (z. B. 'Zahl der Impfpunkte) sollen nachfolgende Notizen gestatten, die bei den jeweilig Pirquetierten gemacht wurden:

Fall Schr: stark sezernierende Fistel der r. Brustwand nach dreimaliger Resektion der r. kariösen VII. bis XI. Rippe '(1908 u. 1909), 1906 Pleuritis sicc. dextra.

Therapie: ambulante Tuberkulinimpfungen.

Am 10. XI. 09: v. P. 2 Punkte am r. Oberarm; 27. XI, : v. P. 2 am r. Oberarm; 3. XIL. 09: v. P. 3 am r. Oberarm; 9. XlI.: v. P. 4 am l. Oberarm; 13. XII.: v. P. 8 an den O.-Armen; 17. XII. 09: v. P. 7 am r. Oberarm; 21. XII.: v. P. 9 auf der Brust; 24. XII.: v. P. 9 an den U.-Armen; 28. XII. 09: v. P. 28 am Bauch, an 29. XII. Schwindel, Müdigkeit, kein Fieber; 3. I. 10: v. P. 84 am Bauch, am 4. XII. elend, stärkere Sekretion der Fistel; 10. I. 10: v. P. 60 am Rücken: 13. I : v. P. 56 am Rücken; 17. I.: v. P. 60 am Rücken; 25. I. 10 : v. P. 46 am Bauch; 29. I.: v. P. 90 am Rücken.

Fall Schä I: Tuberculosis pulmonum: R. Spitzenaffektion, L. Lunge: Kavernenbildung.

Therapie: Tuberkulinimpfungen.

Am 15. XII. 09: v. P. 2; 17. XII.: v. P. 8; 26. XII.: v. P. 21; 2. I. 10 : v. P. 38, am 4. I. Stiche in d. I. Lunge; 8. I. 10: v. P. 80, am 9 . I. Stiche in d. 1. Lunge; 14. I.: v. P. 64 , am 15 . I. morgens $39,5^{\circ}$; 24 . I. 10 : v. P. 57 , am 26. I. Frost, Stiche in d. I. Lunge; 29. I.: v. P. 63, am 31. I. Kopfschmerzen; 11. II. 10 : v. P. 10 ; 19. II.: v. P. 10; 3. III.: v. P. 2 ; 11. III. v. P. 2 ; 24. III.: v. P. 3 .

Fall He: Tuberculosis pulmonum: Bds. Spitzenaffektion, bes. links.

Am 26. XII. 09: v. P. 21, abends $37,5^{\circ}$ (sonst unter $37,2^{\circ}$ ), am 27. XII.: Müdigkeit im Oberkörper, am 28. XII.: stark. Jucken; 6. I. 10: v. P. 57, am 7. I.: Müdigkeit, Kopfschmerzen; 19. I.: v. P. 60, am 20. I.: Müdigkeit, Kopfweb; 29. I. 10 : v. P. 74 ; 3. II.: v. P. $48 ; 11$. II.: v. P. 16; 19. II.: v. P. 10; 4. III.: v. P. 5 ; 12. III.: v. P. 5.

Fall Mi: Karies der Brustwirbel mit Pottschem Gibbus, Senkungsabszess der r. Leistenbeuge.

Therapie: Rauchfusssche Schwebe, Absaugen des Abszesses und Injektion von Jodoformöl. 
Am 27. I. 10: v. P. 2; 29. I.: v. P. 21, am 30. I.: Druck auf der Brust; 6. II.: v. P. 27 , am 7. II. morgens $38,6^{\circ}$, abends 37,6 (sonst nie über $37,3^{\prime \prime}$ ), Hitze, Schwitzen, Durst, Schlafsucht, Schmerz im Gibbus; 12. II. 10: v. P. 10, am 13. II.: Ziehen im Rücken; 17. II.: v. P. 15, am 20. II. Aufflammen der letzten Punkte, Schwitzen; 24. II. 10: v. P. 20, Aufflammen der letzten Punkte; 4. III.: v. P. 25, am 5. III. : Kopfschmerz, am 6. III. Ziehen im Rücken und Leib, Aufflammen; 11. III.: v. P. 25 ; 27. III.: v. P. 7.

Fall Schl: seit dem 10. Jahr Lymphadenitis multiplex tuberculosa colli sin. Therapie: Exkochleation einiger oberflächl. Drüsen, Impfungen.

Am 21. XI. 09: v. P. 2 am Halse 1.; am 22. XI.: Papel $20 \mathrm{~mm}$, hirsekorngrosse, gelbe Bläschen, schmerzhafte Schwelig. am Halse 1.; 15. XII.: v. P. 5 auf d. Brust, am 16. XII.: morgens $37,1^{\circ}$, abends $38^{\circ}$ (sonst weniger), Papel ca. 5-Markstück gross, Kopfschmerz, Appetitlosigkeit; 27. X1I. 09: v. P. 35 auf d. Leib, am 28. XII. abends $37,7^{\circ}$, Kopfschmerz, Appetitlosigkeit; 10. I. 10: v. P. 70 auf d. Leib, am 11. I. morgens $37,6^{\circ}$, abends $38,1^{\circ}$, Appetitlosigkeit, Kopfschmerz, Halsschwellg. 1. (um ca. $4 \mathrm{~cm}$ ); 24. I. 10: v. P. 52 auf d. Leib, am 25. I. morgens $38,3^{\circ}$, abends $37,2^{\circ}$, sonst wie am 11. I., ferner Aufflammen der letzten Papeln; 1. If. 10: v. P. 40 auf d. Leib, am 2. II. morgens $38,6^{\circ}$, abends $37,4^{\circ}$, Schlaf schlecht; 8. II. 10 : v. P. 30 am l. Oberarm; 17. II. : v. P. $15 \mathrm{am}$ 1. U.-Arm, am 18. II.: Appetitlosigkeit, Armschwellg.; 25. II. 10: v. P. 15.

Fall We: Coxitis taberculosa dextra mit Fistel- und Abszessbildung. Therapie: Impfungen. Extensionsverband.

Am 7. XII. 09 : v. P. 4 auf d. Leib; 15. XII.: v. P. 5 auf d. Leib; 23. XII.: v. P. 9 auf d. Leib; 2. I. 10 : v. P. 23 auf d. Leib; 14. I.: v. P. 49 auf d. Leib; 24. I.: v. P. 34 anf d. Leib u. Bein, Punkte auf d. Leib wenig, am r. O.-Schenkel stark gerötet; 1. II.: v. P. 35, Appetit besser, Kind lebhafter, Pals 140; 8. II.: v. P. 27 auf d. Brust, am 9. II. abends $39,7^{\circ}$; 17. II.: v. P. 15 auf r. Bein: 12. III.: v. P. 6 auf r. Arm.

Fall $\mathrm{Pr}$ : Chronische Tuberkulose der linken (und rechten?) Niere.

Am 24. I. 10: v. P. 48 , am 25. I. abends $38,1^{\circ}$ (sonst $37,1^{\circ}$ ), stechender Schmerz in der 1. Nierengegd.; 30. I. 10: v. P. 45 , am 31. I. morgens $38,8^{\circ}$, abends 38,6, Frost, Kopfschmerz, Stechen in der r. u. 1. Nierengegend; 5. II. 10 v. P. 49 , am 6. II. morgens $39,3^{\circ}$, abends $37,5^{\circ}$ (sonst subfebril), Beschwerden wie am 31. I.; 12. II. 10 : v. P. $10 ; 17$. Il. 10 : v. P. 15; 24. II.: v. P. 20 ; 4. III. 10 : v. P. 25, am 5. III. Müdigkeit, Kopfweh, Schmerz in der l. Seite und r. Schenkelbeuge, am 9. III. abends $38,0^{\circ} ; 12$. III. 10 : v. P. 20 , am 13. III.: Schmerz in der l. Nierengegend und r. Schenkelbeuge, rechtsseitige Inguinaldrüsenschwellung; 24. III.: v. P. 7 .

Fall Schrö: Tabes mesaraica.

Therapie: Impfungen.

Am 27. XII. 09: v. P. 49; 14. I. 10: v. P. 54; 24. I. 10: v. P. 48 auf d. Leib, am 25. I. morgens $37,5^{\circ}$ (sonst niedriger), abends $37,6^{\circ}$, Kopfschmerzen; 1. II. 10 : จ. P. $43 ; 8$. II.: v. P. 30 aufs r. Bein; 25. II. 10 : v. P. 10 auf d. r, Arm; 12. III. 10 : v. P. 6.

Fall Bu: Gonitis tuberculosa sinistra mit Abszessbildung. Therapie: Inzision, Jodoform, Streckverband, Impfungen.

Am 24. I. 10: v. P. 2 auf d. Leib; 1. II. 10: v. P. 10 nahe d. l. Knie; 9. II. 10 : v. P. 20 auf d. Leib; 17. II. 10 : v. P. 20 desgl.; 25 . II. 10 : v. P. 15 
am 1. Knie; 8. III. 10: Inzision des Abszesses; 12. III. 10: v. P. 6 am 1. Bein, Papel stärker wie früher; 27. III. 10: v. P. 7 am r. O.-Schenkel.

Fall Schre: Caries ossis pubis dextra recidiva.

'Therapie: Exkochleation, Impfungen.

Am 28. XII. 09: v. P. 32, am 29. XII. morgens $38,6^{\circ}$, abends $38,2^{\circ}$ (sonst unter $37,5^{\circ}$ ), etwas Frost, Kopfschmerz, Appetitlosigkeit, Zieben im Rücken; 10. I. 10 : v. P. 35 , am 11. I. morgens 38,4 , abends $37,2^{\circ}$, Kopfschmerz, Schwitzen, allgemeines Jucken; 19 . I. 10 : v. P. 61 , am 20 . I. morgens $38,3^{\circ}$, abends $38,2^{\circ}$; 29. I. 10 : v. P. 63 , an 30. I. morgens $38,2^{\circ}$, abends $37,7^{\circ}$, zur ambul. Behandlung entlassen; 3. Il, 10: v. P. 49, am 4. II. Ziehen im Rücken; 13. II. 10: v. P. 10.

Fall La: Gonitis tuberculosa sinistra. Caries femoris et tibiae dextrae.

Thera pi e: Chlorkalkbäder, Impfungen.

Am 29. I. 10: v. P. 2 auf d. Bauch; 31. I. 10: v. P. 10, am 1. II.: allg. Schwäche, starke Schweisse; 10. II. 10 : v. P. 15 , 11. II. morgens $38,2^{\circ}$, abends $38,9^{\circ}$ (sonst unter $37^{\circ}$ ), Schwitzen, Kopfschmerz, Appetitlosigkeit, Schmerzen in den Gelenken; 24. II. 10: v. P. 15, am 25. II. morgens $39,2^{\circ}$, abends $38,4^{\circ}$, Schüttelfrost, Auftammen der letzten Reaktionspapeln; 10. III. 10: v. P. 10, Aufflammen, Auftreten gelblicher Bläschen; 24. III. 10: v. P. 6, am 25. III. morgens $37,8^{\circ}$, Kranz gelber Pusteln um die Reaktion. r. Hand.

Fall N: Chronische Entzündung des r. Handgelenks nach Sturz auf die

Therapie: Ruhigstellung, Stauung, Impfungen.

Am 10. 1I. 10: v. P. 2 auf r. U.Arm, am 11. u. 12. III.: Papel $7 \mathrm{~cm}$ breit, roseähnlich, Schwindel, Druck im Kopf; 17. II. 10: v. P. 3 auf r. U.-Arm, am 18. II.: Impfpunkte stark geschwollen, mit roten Ausläufern, mittags $39,5^{\circ}$. Spannung im r. Handgelenk, schlechtes Allgemeinbefinden; 25. II. 10: v. P. 3, Wiederaufflammen der letzten Impfpunkte, leichte Lymphangitis; 5. III. 10: v. P. 3 nahe der Gelenklinie; 12. III. 10: v. P. 3, Wiederaufflammen; 26. III. 10: v. P. 3 auf die Aussenseite der r. Ulna, in die Nähe der Impfpunkte vom 17. II. 10, am 26. III. nachts Frost, starke Rötung und Schwellung des r. Unterarms, Wiederaufflammen der Papeln vom 17. II. und 12. III. 10, heftige Lymphangitis mit Schmerzen in der r. Axilla.

Fall Pic: Emphysem mit chronischer Bronchitis, Bronchialdrüsentuberk.

Am 29. XII. 09: v. P. 2 auf der Brust, Papel $25 \mathrm{~mm}$ am 30. XII.; 10. I. 10 : v. P. 30 , am 11. I. morgens $38,4^{\circ}$, abends $39,1^{\circ} ; 24$. I. 10 : v. P. 49 , am 25 . I. morgens $38,6^{\circ}$, Wiederaufflammen, Kopfweh; 3. II. $10:$ v. P. 25 , am 4. II. Temp. $37,8^{\circ}$, Wiederauffammen; 11. II. 10: . P. 5, Papel $18 \mathrm{~mm}$ am 12. II.; 19. II.: v. P. 10, am 20. II. Papel $25 \mathrm{~mm}$.

Fall Pie: Tuberculosis pulmonum des r. O.- und I. U.-Lappens.

Therapie: Impfungen.

Am 25. I. 10: v. P. 2 ; 2. II. 10 : v. P. $10 ; 11$. III. 10 : v. P. 15 , am 12. II. abends $38,3^{\circ}$, am 13 . II. $38,4^{\circ}$, Impfung ausgesetzt wegen wiederholter Hämoptoe; 24. III. 10: v. P. 4, am 26. III. Papel $15 \mathrm{~mm}$.

Fall Schä II: Tuberculosis pulmonum apic. sin. Diabetes insipidus.

Therapie: Impfungen.

Am 15. II. 10: v. P. 3 ; 18. III.: v. P. 10 , am Abend $37,0^{\circ}$ (sonst geringer), Abgeschlagenheit, Kopfschmerzen, am 20. II. zur ambulanten Behandlg. entlassen; 
am 26. II.: v. P. 10, keine Beschwerden; wegen Periode 14 Tage Pause; 12. III. 10: v. P. 10 .

Fall Fric: Tuberkulose der rechten Niere mit Fistelbildung nach Karies der Wirbelsäule.

Therapie: Spaltang der Fistelgänge, Impfungen.

Am 28. I. 10: v. P. 2; 29. I. 10: v. P. 30, am 30. I.: Kopfweh, Müdigkeit, Schmerz in der l. Seite und an den Wundrändern; 6. II. 10: v. P. 30, am 7. II. Müdigkeit, Urindrang.

Fall Ber: Bronchitis chronica suspecta.

Ther a pie: Impfungen.

Am 8. II. 10: v. P. 2, am 9. II. abends $37,0^{\circ}$ (sonst weniger', Papeln $20 \mathrm{~mm}$; 10. II. 10 : v. P. 10 , am 11. II. morgens $37,0^{\circ}$, Müdigkeit; 18. II. I0: v. P. 15 , am 18. II. abends $37,0^{\circ}$, am 19. II. morgens $37,5^{\prime \prime}$, mittags $38,4^{\circ}$, abends 37,9, Kopfschmerz, Müdigkeit, Appetitlosigkeit.

Fall Fri: Tuberculosis pulmonum des r. Mittel- und 1. U.-Lappens.

'T he rapie: Impfungen.

Am 3. II. 10: v. P. 2; 10. II. 10: v. P. 10, am 11. II. abends $37,2^{\circ}$ (sonst geringer), Papel $6 \mathrm{~mm}$; 19. II. 10: v. P. 15, am 20. Il. abends $37,2^{\circ}$.

Fall BIi: Bronchialdrüsentuberkulose.

Therapie: Impfungen.

Am 25. I. 10: v. P. 2, Reaktion vom 10. II. verschwunden; 21. II. 10: v. P. 5, 22. II. morgens Kopfschmerzen; 24. III. 10: v. P. 3 auf d. Oberarm.

Fall Bu: Tuberculosis pulmonum apicis sin.

The rapie: Impfungen.

Am 16. III. 10: v. P. 2, am 18. III. Puls: morgens 104, abends $108\left(37,0^{\circ}\right.$ u. $\left.37,4^{9}\right)$; 24. III. 10: v. P. 3 am l. Oberarm; 25 . III.: Papel über $20 \mathrm{~mm}$.

Fall Gr: Tuberculosis pulmonum apicis sin.

The r a pie: Impfungen.

Am 26. II. 10: v. P. 3 auf der Brust; 12. III. 10: v. P. 5 auf der r. Schulter.

Fall Sch ä 1II: Skrofulose.

The rapie: Impfungen (ambulante).

Am 26. II. 10: v. P. 5, Papeln nach 2 Wochen noch über $20 \mathrm{~mm}$ breit, mit hirsekorngrossen Pusteln besetzt; 12. III. 09: v. P. 10.

Fall Ne: Tuberculosis pulmonum apicis dext. sin.

Therapie: Impfungen.

Am 12. III. 10: v. P. 15 , am 13 . III. morgens $37,6^{\circ}$, abends 37,4 (sonst weniger), Ziehen in der Brust, Kopfschmerzen; 25. III. 10: v. P. 3.

Fall Wv: Tuberculosis pulmonum des rechten Unterlappens.

Ther a pie: Impfungen.

Am 19. III. 10: v. P. 2 am r. O.-Arm, nachts Schlaflosigkeit, Schwitzen, am 20. III. morgens $37,2^{\circ}$, abends $37,0^{\circ}$ (sonst unter 37), Papel bis $19 \mathrm{~mm}$ breit; 29. III. 10 : v. P. 3 am 1. O.-Arm, abends $37,0^{\circ}$, am 30. III. $37,2^{\circ}$, Puls 120 abends, starke Kopfschmerzen, Papel 25 bis $35 \mathrm{~mm}$ breit.

Wie aus der Betrachtung der einzelnen Versuchsanordnungen erhellt, wurden die therapeutischen Impfungen im allgemeinen gut 
vertragen. Fieber, Allgemeinerscheinungen und Herdreaktionen traten erst dann auf, wenn mehr als 10 Impfpunkte angelegt wurden. Besonders war dies bei schroffer Steigerung der Punktzahl der Fall. Die positiv Reagierenden liessen hinsichtlich dieser Erscheinungen insofern einen Unterschied untereinander erkennen als solche I. Grades viel höhere Impfpunktzahlen reaktionslos ertrugen, wie diejenigen II. Grades. Bei einigen Patienten mit hoher Überempfindlichkeit genügten schon 2 bis 3 Impfpunkte, um Schwindel, Kopfschmerzen und leichtes Frösteln herrorzurufen. Jarosch hat ebenfalls eine Beobachtung mitgeteilt, die zeigt, dass es bei der von Pirquetschen Kutanimpfung zu einer starken Allgemeinreaktion (Störung des Allgemeinbefindens, Temperatursteigerung, Lokalreaktion auf den Lungen) kommen kann. Gewöhnlich klagten unsere Pirquetierten beim Eintritt von Allgemeinerscheinungen über Abgeschlagenheit, Kopfweh, Gliederschmerzen, Appetitlosigkeit, Schwitzen, Hitze, Durst und Frösteln. Die Temperatursteigerungen erreichten niemals $40,0^{\circ}$ C. Herdreaktionen äusserten sich je nach dem Krankheitssitz verschieden. Die befallenen Lungenpartien antworteten mit Bruststichen, vermebrtem Husten und Auswurf.

Objektiv liess sich vermehrtes Rasseln feststellen, in einem Falle war mir dies schon nach Impfung mit 3Punkten möglich. Es handelte sich um einen Kranken mit einem zirkumskripten Herd des rechten Unterlappens. Einmal beobachtete ich rezidivierende Hämoptoe, die jedoch auch trotz längerer Impfpause immer von neuem sich einstellte, also wohl nicht durch die Kutanreaktion bedingt war. Bei Nierentuberkulose traten stechende Schmerzen in der erkrankten Niere, Urindrang, vermehrte Albuminurie auf; bei tuberkulösen Fisteln erhöhte Eitersekretion sowie Schmerz an den Wundrändern; bei Knochenkaries ziehende Schmerzen im Krankheitsherd z. B. im Pottschen Gibbus; bei tuberkulösen Lymphdrüsenaffektionen beträchtliche Schwellung der erkrankten Drüsen (in einem Falle um $5 \mathrm{~cm})$. Bei einer Patientin mit Nierentuberkulose trat wiederholt eine deutliche Anschwellung der rechtsseitigen Leistendrüsen auf, einerlei, ob die Impfung in der Nähe oder weit entfernt von der Leistenbeuge vorgenommen wurde. Es handelte sich wohl in diesem Falle um eine spezifische Erkrankung der genannten Drüsen. Wolff-Eisner beobachtete zweimal Drüsenschwellungen im Anschluss an die Impfung, deren Auftreten nach seiner Ansicht möglicherweise durch eine besonders heftige Reaktion lierrorgerufen ist, "da die Serumkrankheit, die nach Einverleibung ron körperfremdem Eiweiss auftritt, sich ebenfalls durch Fieber und Drüsenschwellungen äussert." Die eben geschilderten Allgemeinerscheinungen, Temperatursteigerungen und Herdreaktionen ähneln sehr dem Reaktionsbilde, 
das man nach stärkeren Tuberkulininjektionen eintreten sieht, ohne jedoch dessen Stärke zu erreichen. Über die Ursache dieser unliebsamen Begleiterscheinungen der therapeutischen Kutanimpfungen, die keineswegs die Regel bildeten und, wie gesagt, nur unter bestimmten Umständen auftraten, will ich mich weiter unten auslassen.

Von besonders interessanten Reaktionserscheinungen wäre noch das Wiederauflammen alter Impfpapeln zu nennen. Dieses Phänomen beobachtete ich bei 5 Kranken mit grosser Überempfindlichkeit. Stets flammten die zuletzt gesetzten Impfpunkte auf, während die älteren höchstens eine geringe Rötung zeigten.

Eine starke Lymphangitis mit Drüsenschwellung trat wiederholt bei einer Geimpften am Reaktionstage auf, ohne dass etwa mit unsauberem Impfbohrer oder Tuberkulin gearbeitet worden war.

Die Ergebnisse der Kutanimpfungen waren folgende:

Lnter Berücksichtigung des gesamten Entwickelungsbildes der Krankheit, einschliesslich der subjektiven Beschwerden, war ein positiver Erfolg in 21, ein negativer in 3 Fällen zu konstatieren. Bemerken möchte ich schon an dieser Stelle, dass mir leider ein zu kleines Material und eine zu kurze Beobachtungszeit zur Verfügung. stand, um ein endgültiges Urteil über den Wert kutaner Tuberkulinimpfungen zu fällen. Indes war gerade der persönliche Eindruck von dem günstigen Einfluss der Kutanreaktion auf tuberkulöse Prozesse ein derartiger, dass ich mich zur Publikation meiner Erfahrungen berechtigt glaubte, zumal mir die Fortsetzung der Untersuchungen nicht mehr möglich ist.

Der heilsame Einfluss der Impfungen machte sich bei den Kranken zuerst bemerkbar durch Hebung des Allgemeinbefindens. Schlaf und Appetit wurden sichtlich besser. Kopfschmerzen, Schwindel und Abgeschlagenbeit wurden bald geringer. Die Nachtschweisse verschwanden. Das Körpergewicht nahm zu. Die Menge der Tuberkelbazillen im Sputum etc. wurde kleiner. Bei einzelnen Patienten waren überhaupt keine mehr zu finden. Am spätesten kehrte die febrile Temperatur zur Norm zurück. Die Änderung des objektiven Krankheitsbefundes war je nach dem Sitz des Prozesses eine verschiedene. Bei Lungenkranken zeigte sich eine Abnabme der Dämpfung und Rasselgeräusche, in einigen Fällen sogar ein völliges Verschwinden derselben. Drüsen- und Gelenkschwellungen liessen eine deutliche Differenz des Umfanges der betroffenen Partien zu Beginn und Ende der Kur erkennen. Tuberkulöse Fisteln schlossen sich. Bei sämtlichen Phthisikern I. Stadiums verschwand das Fieber, desgleichen die Rasselgeräusche mit Ausnahme einer Patientin, die eine doppelseitige Spitzenaffektion, besonders links hatte. 
In diesem Falle hörte man noch RVO und RHO bisweilen Spuren von zähem Rasseln, wiederum stärker LVO und LHO. Die Lungenkranken II. Stadiums zeigten gleichfalls einen Rückgang der Temperatur zur Norm, jedoch kein völliges Verschwinden des Rasselns. Ein Lungentuberkulöser III. Stadiums, dessen linke Lunge von oben bis unten rasselte und alle Kavernensymptome aufwies [Röntgenbild: L. Lungenfeld vollständig verdunkelt, nur im 1. I. I.-R. eine fünfmarkstückgrosse helle Stelle $=$ Kaverne], über dessen rechter Spitze spärliches, leicht klingendes Rasseln zu hören war, liess ein Verschwinden des rechtsseitigen Spitzenbefundes nach 13 maliger Impfung erkennen. Die Kaverne der 1. 1. I.-R. war im Röntgenbild nicht mehr sichtbar, im übrigen war der Status derselbe wie früher. Dieser Kranke hatte zuvor sogar positive Diazoreaktion im Úrin. Merkwürdig war ein $\mathrm{Fall}$ mit beginnender Spitzenaffektion, der mit einem typischen Diabetes insipidus rergesellschaftet war. Die bis dahin vergeblich behandelte Kranke blühte nach 4 maliger Impfung sichtlich auf. Die Urinmenge ging betrüchtlich zurück. Die besten Resultate zeitigte die kntane Tuberkulinimpfung bei Lymphdrüsenaffektionen und suspekter chronischer Bronchitis. Bei einem Patienten mit tuberkulösen Drüsen an der linken Seite des Halses waren die Drüsenpakete nach $3^{1 / 4}$ monatiger Behandlung um die Hälfte kleiner geworden.

Zwei Patientinnen mit suspekter chronischer Bronchitis, die beide hereditär belastet waren und sehr stark auf die diagnostische v. Pirquetsche Impfung reagiert hatten, zeigten nach einer Reihe von Impfungen ein völliges Verschwinden der sehr hartnäckigen Erkrankung. 2 Nierentuberkulöse und ein Kind mit allgemeiner Drüsentuberkulose, besonders der Mesenterialdrïsen, Meteorismus, häufigen Diarrhöen machten keine Fortschritte. Nur der Crinbefund änderte sich bei der einen Nierenkranken insofern, als trotz eifrigsten Suchens keine Tuberkelbazillen mehr nachweisbar waren.

Sehr erfreuliche Resultate erzielte ich auch bei einigen Patientenmit Knochenkaries. Auffallend war die Besserung, die bei einem ganz kachektischen 31 jährigen Patienten mit doppelseitiger Gonitis tuberculosa und Knochenkaries nach 6 Impfungen zu konstatieren war. Dieser Kranke hatte bei seiner Aufnahme am rechten Knie und Unterschenkel etwa 14 stark eiternde Fisteln. Nach Ablauf ron etwa $1^{1 / 3}$ IIonaten waren 6 Fisteln geheilt. Das Allgemeinbefinden war ein rorzügliches. Die Gesichtsfarbe wurde frisch. Das Körpergewicht nahm um 7 Pfund $400 \mathrm{~g}$ zu. Ein anderer Patient, dem wegen Rippenkaries die VII. bis XI. rechte Rippe reseziert worden war, nahm während der 21/2 monatigen Impfliur um etwa 20 Pfund zu. Vor der 
vorher bestehenden Fistel, die 2 Seitengänge hatte, war nur noch ein zirka $5 \mathrm{~cm}$ langer, schmaler Gang übrig. Das Aussehen des bis dahin recht elenden Mannes wurde ein so gutes, dass es Angehörigen und Bekannten auffiel. Einen schönen Erfolg beobachtete ich weiterhin bei einer kachektischen Frau mit Karies der Brustwirbelsäule, Gibbus Pottii und rechtsseitigem Senkungsabszess. Hebung des Allgemeinbefindens, Abnahme der Druckempfindlichkeit des Gibbus, Körpergewichtszunahme um 8 Pfund 350 Gramm in $1^{3 / 4}$ Monaten waren zu verzeichnen. Diese Beispiele mögen genügen, um die Wirkung der therapeutischen Impfung zu veranschaulichen. Hinsichtlich der Dauerresultate möchte ich bemerken, dass sich von den längere Zeit Pirquetierten kürzlich 3 zur Nachkontrolle rorstellten. Bei allen 3 hielt erfreulicherweise die Besserung an. Auch das Gewicht hatte weiter zugenommen. Was endlich die Gewichtszunahme betrifft, so war sie im allgemeinen um so höher, je länger die Kranken der Behandlung unterstanden. Nicht zugenommen hatten zwei Patienten. Dass die Gewichtszunahme nicht allein auf den Krankenhausaufenthalt mit seinen besseren hygiєnischen Verhältnissen etc. zurückzuführen war, dafür spricht einmal die Tatsache, dass auch ambulant Pirquetierte zum Teil recht beträchtlich an Körpergewicht gewannen, andererseits die Beobachtung, dass die Kranken vielfach erst im Anschluss an die Kutanreaktionen Körpergewichtszunahme zeigten.

Über das Zustandekommen der v. Pirquetschen Reaktion bestehen mehrere Anschaungen. Man stellt sich vor, dass sämtliche Zellen des tuberkulös infizierten Organismus stets Schutzstoffe, Antitoxine, in grösserer Menge bilden, als zur Bindung der Toxine nötig sind. Werden nun ron aussen Toxine eingeführt, so werden die im Körper überschüssig vorhandenen Schutzstoffe sofort gebunden.

Nach v. Pirquet und Wassermann kommt es bei dem Zusammentreffen ron Toxin und Antikörper zu einer lokalen Gewebsreizung, bei der die Leukozyten eine hervorragende Rolle spielen.

Wolff-Eisner meint, dass die Tuberkulinreaktion nicht im Krankheitsherde zustande kommt, sondern dass unter dem Einfluss der Tuberkelbazillengifte der ganze Körper in dem Zustande der Reaktionsfähigkeit mit und gegen das Tuberkulin sich befindet.

Bei den pathologisch-histologischen Befunden der Papel, wie sie Wolff-Eisner, Bandler und Kreibich, Dach und insbesondere $\mathrm{Zi}$ ieler beschrieben haben, ist zweierlei auffällig: Einmal das Auftreten von typischen Epitheloidzellentuberkeln mit starken Lymphozytenanhäufungen im Verlauf der Gefässe, andererseits die lange Dauer der histologischen Veränderungen wie sie gerade Zi eler be- 
obachtete (Zunahme der Veränderungen in den ersten 3 bis 5 Wochen nach der Tiefe und in die Breite, Vorhandensein selbst noch nach $3^{1 / 2}$ Monaten).

Lymphozytenansammlungen im Gefolge tuberkulöser Affektionen sind so etwas Alltägliches, dass man das Vorkommen derselben in Exsudaten, Spinalflüssigkeit, Sputum; Urin direkt zur Frühdiagnose der Tuberkulose verwandte. Eine experimentell sichere Erklärung für die genannte Erscheinung besitzen wir leider nicht. Vielleicht haben uns die interessanten Ergebnisse der Bergel schen Versuche in der Erforschung dieser wenig geklärten Fragen einen Schritt rorwärts gebracht. Nach $\mathrm{Hammerschlag}$, Ruppel und Sata enthält der Tuberkelbazillus eine fetthaltige Substanz. Bergel glaubt nun auf Grund seiner Experimente annehmen zu dürfen, dass die Lymphozyten ein fettspaltendes Ferment enthalten. Nach seiner Auffassung ist die reaktive Ansammlung von Lymphozyten um den Erkrankungsherd das Resultat der chemotaktischen Reizwirkung zwischen den fetthaltigen Tuberkuloseerregern und fettspaltenden einkernigen, weissen Blutkörperchen.

Ist es schon schwer, eine Erklärung des Zustandekommens der v. Pirquetschen Reaktion zu geben, so dürfte dies erst recht zutreffen für die Frage des Mechanismus der sogenannten Heilwirkung kutaner Tuberkulinimpfungen. Nach meiner Auffassung ist ein Teil der im Körper des Geimpften sich abspielenden Vorgänge etwa folgendermassen zu denken:

Was zunächst die rasch wieder verschwindende Flächenröte betrifft, so handelt es sich wohl um ein Vasomotorenphänomen, hervorgerufen durch starke Erregung vasodilatatorischer Bahnen. Nach M o r e s Auffassung besteht bei tuberkuloseinfizierten Individuen eine spezifische (der Tuberkulose eigentümliche) Reizbarkeit des Nervensystems gegenüber dem Tuberkulin, sozusagen eine spezifische nervöse "Allergie" (veränderte Reaktionsfähigkeit). Diese scheint nach meinen Beobachtungen um so grösser und anhaltender zu sein, je reaktionsfähiger der Geimpfte ist. Die Rötung an der Impfstelle selbst, die nach dem Verschwinden dieses öfters Erysipel ähnlichen Hauterythems allmählich einsetzt, mag wohl zunächst zum Teil seinen Grund ebenfalls in der durch das eindringende Tuberkulin bedingten Vasomotorenerregung haben. Die bei gut Reagierenden schon nach 24 bis 48 Stunden fühlbare Papel scheint durch die nun einsetzende Lymphozyteninfiltration verursacht zu sein.

Durch die von Pirquet sche Impfung ist gleichsam ein künstlicher Tuberkuloseherd auf der Haut geschaffen, nur dass dieser zum Unterschied von echter Tuberkulose der lebenden, spezi- 
fischen Erreger entbehrt, die unaufhörlich mit ihrem Gift neue Zellkomplexe vernichten, ein Unterschied, der uns auch den weiteren Verlauf verstehen lässt. Sobald die Lymphozyten den vermeintlichen Feind überwunden haben, wenden sie sich nach dieser Irreführung dem wirklichen Tuberkuloseherd zu, um dort den L ymphozytenwall, der durch die fortgesetzte Toxinabsonderung des Tuberkels gelähmt oder gegen Reize abgestumpft ist, zu verstärken. Je frischer die Tuberkuloseinvasion ist, um so rascher tritt anscheinend der genannte Mechanismus in Aktion. Das Wiedera ufflammen der alten Papeln erklärt sich damit, dass bei solchen Kranken, die, wie gesagt, stets stark reagiert hatten, die Kutanreaktion nur dem äusseren Anschein nach verschwunden war, während in Wirklichkeit der Kampf in der Ti efe noch weiter tobte.

Spätreaktionen, die im allgemeinen bei abgeheilten Prozessen (z. B. verkalkte Drüsen) vorkommen sollen, weisen m. E. darauf hin, dass die geschilderten Vorgänge nur langsam in Erscheinung treten, wofür auch die histologischen Befunde sprechen. Der Körper scheint im erfolgreich durchgeführten Kampfe an der Grenze seiner Leistungsfähigkeit angekommen zu sein. Es ist ja eine tagtäg. liche Erscheinung, dass die Tuberkulose, je länger sie dauert und je weiter sie fortschreitet, ganz im Gegensatz zu den meisten anderen Infektionskrankheiten, keine $\mathrm{Zu}$-, ja vielmehr Abnahme der Schut kräfte zeigt. Inwieweit Allgemeinerscheinungen, Herdreaktionen sowie Temperatursteigerungen bei kutanen Tuberkulinimpfungen durch die eigentliche Pirquetsche Reaktion oder durch die Resorption von Tuberkulin bedingt sind, ist schwer zu sagen. Jedenfalls kann man wohl annehmen, dass das resorbierte Tuberkulin durch die Passage der verschiedenen Hautschichten einen wesentlichen Teil seiner giftigen Eigenschaften eingebüst hat, während dies bei dem injizierten Tuberkulin nicht der Fall sein dïrfte. Nach Wolff-Eisners Ansicht, die ich erst vor einigen Tagen kennen lernte, werden durch intrakutane Injektionen kleiner Tuberkulindosen ( $1 / 10-1 / 1000 \mathrm{mg})$ und Einreibung von Tuberkulinsalbe $(20-50 \%$ ) künstlich Rezeptoren in indifferenten Gewebsteilen (z. B. Bindegewebe) geschaffen, welche Tuberkulin an sich ziehen und die Giftwirkung lokalisieren. Die soeben von mir entwickelte Auffassung vom Zustandekommen der Kutanreaktion und ihrer therapeutischen Bedeutung will nur einen der Momente erklären, die eine Rolle bei der Impfung spielen dürften. Aller Wahrscheinlichkeit nach bilden sich auch Antikörper gegen das Koch sche Alttuberlkulin, ob auch gegen das im Körper produzierte Tuberkelbazillengift, ist eine andere 
Frage, die noch der Lösung harrt. Es entstehen im tuberkulösen Körper höchst wahrscheinlich schon durch den Prozess an sich Schutzkörper. Der tuberkulöse Organismus ist ja keineswegs arm an Zerfallsprodukten.

Die Tuberkulinempfind lichkeit $n$ i mmt mit dem wachsenden Umfang des tuberkulösen Prozesses bis zu einer gewissen $\mathrm{Grenzezu}$, deren Festlegung noch unmöglich ist. $\mathrm{Ob}$ wir mittelst der Komplementablenkungsmethode Aufschluss über die Frage der Schutzkörperbildung bei Tuberkulösen bekommen, wird die Zukunft lehren.

L üdke konnte mittelst dieses Verfahrens spezifische Reaktionsprodukte im tuberkulösen Serum nachweisen, die am häufigsten im Blutserum der mit Tuberkulin injizierten Phthisiker zu finden waren. Beziehungen zwischen dem Antituberkulinnachweis und der Schwere der Infektion bestehen nach seiner Auffassung nicht. Er sagt: Es können diese Reaktionssubstanzen nicht als Antikörper mit antitoxischen oder antiinfektiösen Qualitäten bezeichnet werden; denn bei einem deutlich nachweisbaren Antituberkulingehalt des Blutserums kann die Erkrankung fortschreitenden Charakter tragen und in anderen Fällen, in denen sich kein Antituberkulingehalt nachweisen liess, zeigte die Krankheit merkliche Heilungstendenz. Nach Cohn bedingt der Antituberkulinnachweis des Blutes durchaus nicht immer eine geringere Empfindlichkeit gegenüber Tuberkulin.

Köhler hebt ausdrücklich hervor, dass hoher Antikörpergehalt sowie Immunität gegenüber Tuberkulin keineswegs identisch ist mit Immunität gegenüber Tuberkulose. Die Unempfindlichkeit gegenüber Tuberkulin bedeutet nicht geschehene Abheilung eines tuberkulösen Prozesses im Körper.

Die Vorteile der kutanen Tuberkulinimpfungen dürften dem bisherigen Injektionsverfahren gegenüber folg ende sein:

1. sind sie leicht und bequem, olne grössere Vorbereitungen, ausführbar;

2. sind sie bei streng individueller Anwendung vollständig gefahrlos;

3. Wird durch Anlegung künstlicher Giftdepots auf der Haut ein Teil der Reaktionsvorgänge an die Körperoberfläche verlegt, wodurch einerseits ein Einblick in die Reaktionsfähigkeit des Körpers und damit eine streng individuelle Behandlung ermöglicht wird, andererseits die Nachteile, die der Injektionsbehandlung (ev. Propagation des Krankheitsprozesses etc.), auf ein Minimum reduziert sind. 
Als Leitm otiv für die Behandlung Tuberkulöser mit kutanen Impfungen hat geeignete Auswal und vorsichtige, dem Einzelfall genau angepasste Behandlung zu gelten. Geeignet sind vor allem solche Patienten, die auch gegen Tuberkulininjektionen besonders überempfindlich sind (Skrofulöse, an Knochenund Gelenktuberkulose sowie beginnender Phthise Leidende). Es sind dies Kranke mit positiver Reaktion II. Grades. Un geeignet sind solche Tuberkulöse, die trot $z$ wiederholter Impfung keine Zunahme der Reaktionspapel zeigen.

Wichtig für die Behandlung ist die möglichste Vermeidung jeglicher Beeinträchtung des Allgemeinbefindens. Stärkere, schädigende Reaktionen lassen sich durch geeignete Wahl in der Zahl der Impfpunkte umgehen. Die erste v. Pirquet sche Impfung mit 2 Punkten hat einen doppelten Zweck:

1. Soll sie die Tatsache der tuberkulösen Infektion überhaupt feststellen, falls dies nicht durch Bazillennachweis möglich war.

2. Soll die Stufe der Überemptindlichkeit aus dem Grade der Reaktion ermittelt werden.

Um den Sitz und den Charakter einer tuberkulosererdächtigen Affektion zu eruieren, genügt meist nicht die einfache diagnostische v. Pi rquetsche Impfung mit nur 2 Punkten, da der positive Ausfall der Reaktion nach dem 2. Jahre nur besagt, dass das in Frage kommende Individuum einmal tuberkulös infiziert war, dagegen nicht aktive von inaktiver Tuberkulose unterscheiden lässt, geschweige denn die topische Diagnose ermöglicht. Im allgemeinen konnte ich bei stark Reagierenden mit ca. 10 bis 15 , bei mittelmässig Reagierenden mit ca. 25 bis 30 Punkten ein Reaktionsbild erzeugen, das, wie schon erwähnt, demjenigen ähnelt, welches man nach der Kochschen probatorischen Injektion auftreten sieht, ohne jedoch dessen Stärke zu erreichen.

Hat man sich einen genügenden Einblick in den Charakter und Sitz des Prozesses sowie die Reaktionskraft des kranken Organismus verschafft, so kann man zu den eigentlichen therapeutischen Impfungen übergehen. Bei der zweiten Vakzination, die ca. 10 bis 14 Tage nach der ersten erfolgt, kann man drei Impfpunkte setzen. Werden diese ohne irgend welche Störungen des Allgemeinbefindens vertragen, so darf man nach der eben angegebenen Pause wieder um einen Impfpunkt steigen etc. Traten jedoch Erscheinungen einer stärkeren Reaktion (Puls, Temperatur, Allgemeinbefinden!) auf, so muss man warten, bis diese völlig verschwunden sind und in der Impfpunktzahl zurückgehen bezw. nicht höher steigen. Da ich der An- 
sicht bin, dass die Überempfindlichkeit eine Schutzeinrichtung des Erkrankten darstellt, die man nicht durch allzuhäufiges Impfen mit rascher Steigerung der Punktzahl überanstrengen soll, möchte ich raten, im Zweifelsfalle lieber etwas längere Pausen zu machen. Rasches Sinken der Reaktionszeit bei einem Patienten, der bei den ersten Impfungen eine langanhaltende Papel aufwies, halte ich - nach meinen Beobachtungen - für ein prognostisch ungünstiges Zeichen. Ob der Ort der Impfung einen merklichen Eintluss auf den Verlauf der Reaktion hat, ist mir noch unklar. Jedenfalls konnte ich keine Propagation tuberkulöser Prozesse durch Impfungen in der Nähe des Krankheitsherdes bemerken.

Den Gesamteindruck ron der therapeutischen Wirksamkeit kutaner Tuberkulinimpfungen möchte ich unter Berücksichtigung der Schwierigkeiten, die gerade die Tuberkulose für die Beurteilung eines Heilmittels bietet, karz dahin resümieren, dass er im allgemeinen ein recht günstiger war. Ihren wirklichen Wert wird man natürlich erst aus grösseren Beobachtungsreihen ermitteln können als sie mir zur Verfügung standen. In welchen Fällen die Injektionsmethode der v. Pirquetschen Impfung vorzuziehen ist, darüber muss das einzelne Krankheitsbild entscheiden, wie ich rorhin angedeutet habe. Das von mir angegebene Verfahren soll keineswegs bewährte Behandlungsmethoden (z. B. Biersche Staung bei Knochen- und Gelenktuberkulose, Injektion von Jodoformglyzerin etc.) verdrängen, im Gegenteil unser Rüstzeug im Kampfe gegen die schlimmste Volkskrankheit um ein neues, wie ich hoffe, wertvolles Kampfmittel bereichern.

\section{L iteratur.}

1. Berge 1: Fettspaltendes Ferment in den Lymphozyten. Münch. med. Wochenschr. 1909. Nr. 2.

2. H. Jarosch: Beitrag zur Kasuistik der v. Pirquetschen Klutanimpfung. Zeitschr. f. Tuberkulose 1909. Bd. 15. S. 176-178.

3. Köhler: Die therapeutische Beeinflussung der inneren und äusseren Tuberkulose durch 'Tuberkulin und verwandte Mittel. Med. Klinik 1909. Nr. 2.

4. Lïdke: Díe praktische Verwertung der Komplementbindungsreaktion. Münch. med. Wochenschr, 1909. Nr. 26.

5. W. Münch: Die kutane Tuberkulinreaktion nach v. Pirquet. Inaug.-Diss. Giessen 1909.

6. Wolff-Eısner: Die Bedeutung der Konjunktival-Reaktion nach 4000 Beobachtungen. Münch. med. Wochenschr. 1908. Nr. 45.

7. H. Zieler: Experimentelle Untersuchungen über „tuberkulöse ${ }^{\star}$ Veränderungen an der Haut ohne Mitwirkung von Tuberkelbazillen (toxische Tuberkulosen) und die Bedingungen ihres Entstehens, Münch. med. Wochenschr, 1908. Nr. 32. 